\title{
Research on Adjoint Kernelled Quasidifferential
}

\author{
Si-Da Lin, ${ }^{1}$ Fu-Min Xiao, ${ }^{2}$ Zun-Quan Xia, ${ }^{1}$ and Li-Ping Pang ${ }^{1}$ \\ ${ }^{1}$ School of Mathematical Sciences, Dalian University of Technology, Dalian 116024, China \\ ${ }^{2}$ Department of Hydrography \& Cartography, PLA Dalian Naval Academy, Dalian 116018, China
}

Correspondence should be addressed to Si-Da Lin; 1sdsl@163.com

Received 10 October 2013; Accepted 19 January 2014; Published 5 March 2014

Academic Editor: Hichem Ben-El-Mechaiekh

Copyright (C) 2014 Si-Da Lin et al. This is an open access article distributed under the Creative Commons Attribution License, which permits unrestricted use, distribution, and reproduction in any medium, provided the original work is properly cited.

\begin{abstract}
The quasidifferential of a quasidifferentiable function in the sense of Demyanov and Rubinov is not uniquely defined. Xia proposed the notion of the kernelled quasidifferential, which is expected to be a representative for the equivalence class of quasidifferentials. Although the kernelled quasidifferential is known to have good algebraic properties and geometric structure, it is still not very convenient for calculating the kernelled quasidifferentials of $-f$ and $\min \left\{f_{i} \mid i \in\right.$ a finite index set $\left.I\right\}$, where $f$ and $f_{i}$ are kernelled quasidifferentiable functions. In this paper, the notion of adjoint kernelled quasidifferential, which is well-defined for $-f$ and $\min \left\{f_{i} \mid i \in I\right\}$, is employed as a representative of the equivalence class of quasidifferentials. Some algebraic properties of the adjoint kernelled quasidifferential are given and the existence of the adjoint kernelled quasidifferential is explored by means of the minimal quasidifferential and the Demyanov difference of convex sets. Under some condition, a formula of the adjoint kernelled quasidifferential is presented.
\end{abstract}

\section{Introduction}

Quasidifferential calculus, developed by Demyanov and Rubinov, plays an important role in nonsmooth analysis and optimization. The class of quasidifferentiable functions is fairly broad. It contains not only convex, concave, and differentiable functions but also convex-concave, D.C. (i.e., difference of two convex), maximum, and other functions. In addition, it even includes some functions which are not locally Lipschitz continuous. Quasidifferentiability can be employed to study a wide range of theoretical and practical issues in many fields, such as in mechanics, engineering, and economics nonsmooth analysis and fuzzy control theory (see, e.g., $[1-13])$.

A function $f$ defined on an open set $\mathcal{O} \subset R^{n}$ is called quasidifferentiable (q.d.) at a point $x \in \mathcal{O}$, in the sense of Demyanov and Rubinov [5], if it is directionally differentiable at $x$ and there exist two nonempty convex compact sets $\underline{\partial} f(x)$ and $\bar{\partial} f(x)$ such that the directional derivative can be represented in the form as

$$
f^{\prime}(x ; d)=\max _{u \in \underline{\partial} f(x)}\langle u, d\rangle+\min _{v \in \bar{\partial} f(x)}\langle v, d\rangle, \quad \forall d \in R^{n},
$$

where $\langle\cdot, \cdot\rangle$ denotes the usual inner product in $R^{n}$. The pair of sets $D f(x)=[\underline{\partial} f(x), \bar{\partial} f(x)]$ is called a quasidifferential of $f$ at $x$ and $\underline{\partial} f(x)$ and $\bar{\partial} f(x)$ are called a subdifferential and a superdifferential, respectively.

It is well known that the quasidifferential is not uniquely defined. Let $Y_{n}$ be the set of all nonempty convex compact sets in $R^{n}$. Denote $A \pm B=\{a \pm b \mid a \in A, b \in B\}$ and $\lambda A=\{\lambda a \mid a \in A\}$, where $A, B \in Y_{n}$ and $\lambda \geq 0$. Suppose that $[U, V]$ is a quasidifferential of $f$; then, for any $A \in Y_{n}$, the pair of sets $[U+A, V-A]$ is still a quasidifferential of $f$. And the set $\mathscr{D} f(x)$ of quasidifferentials of $f$ at $x$ is so large that the whole space $R^{n}$ could be covered by the union of subdifferentials or superdifferentials; that is,

$$
R^{n}=\bigcup_{D f(x) \in \mathscr{D} f(x)} \underline{\partial} f(x)=\bigcup_{D f(x) \in \mathscr{D} f(x)} \bar{\partial} f(x) .
$$

The quasidifferential uniqueness is an essential problem in quasidifferential calculus, so it is necessary to find a way by which a quasidifferential, particularly a small quasidifferential in some sense, as a representative of the equivalence class of quasidifferentials, can be determined automatically. The problem was for the first time considered in a discussion at IIASA, by Demyanov and Xia in 1984 [4]. There were 
many reports and publications mentioning or dealing with this subject from different points of view (see, for instance, [9-26], etc.).

Pallaschke et al. [18] introduced the notion of the minimal quasidifferential and proved the existence of equivalent minimal quasidifferential. $[U, V] \in \mathscr{D} f(x)$ is called minimal, provided that $\left[U_{1}, V_{1}\right] \in \mathscr{D} f(x)$ satisfying $U_{1} \subset U$ and $V_{1} \subset V$ implies $U=U_{1}$ and $V=V_{1}$. Nevertheless, the minimal quasidifferential is not uniquely defined either. Indeed, any translation of a minimal quasidifferential is still a minimal quasidifferential; in other words, if $[A, B]$ is a minimal quasidifferential, then, for any singleton $\{c\}$, the pair of sets $[A+\{c\}, B-\{c\}]$ is still a minimal quasidifferential. For one-dimensional space, equivalent minimal pairs are uniquely determined up to translations, according to [8]. Grzybowski [15] and Scholtes [22] proved independently the fact that equivalent minimal quasidifferentials, in the two-dimensional case, are uniquely determined up to a translation. For the $n$-dimensional case $(n \geq 3)$, Grzybowski [15] gave the first example of two equivalent minimal pairs in $R^{3}$ which are not related by translations, and, as in [19], Pallaschke and Unbański indicated that a continuum of equivalent pairs are not related by translation for different indices. Some sufficient conditions and both sufficient and necessary conditions for the minimality of pairs of compact convex sets were given and some reduction techniques for the reduction of pairs of compact convex sets via cutting hyperplanes or excision of compact convex subsets were proposed according to Pallaschke and Urbański [20, 21].

For the same purpose, Xia [24, 25] introduced the notion of the kernelled quasidifferential. It was proved that

$$
\begin{aligned}
& S=\bigcap_{D f(x) \in \mathscr{D} f(x)}(\underline{\partial} f(x)+\bar{\partial} f(x)), \\
& \bar{S}=\bigcap_{D f(x) \in \mathscr{D} f(x)}(\bar{\partial} f(x)-\bar{\partial} f(x))
\end{aligned}
$$

are nonempty, according to Deng and Gao [14]. $S$ and $\bar{S}$ (defined by (3)) are called sub- and super-kernel, respectively, and $[S, \bar{S}]$ is called a quasi-kernel of $\mathscr{D} f(x)$. The quasi-kernel is said to be a kernelled quasidifferential of $f$ at $x$ if and only if the quasi-kernel $[S, \bar{S}]$ is a quasidifferential, denoted by $D_{k} f(x)=\left[\underline{\partial}_{k} f(x), \bar{\partial}_{k} f(x)\right]$. If $f$ has a kernelled quasidifferential at $x \in R^{n}$, then $f$ is said to be a kernelled quasidifferentiable function at $x$. For the case of one-dimensional space, the existence of the kernelled quasidifferential was given by Gao [16]. In the two dimensional case, based on the translation of minimal quasidifferentials, it was proved that the kernelled quasidifferential exists for any q.d. function (see [17]). In the $n$-dimensional case $(n \geq 3)$, whether the pair of sets given in (3) is a quasidifferential of $f$ at $x$ is still an open problem, some progress has been made in the last years. Zhang et al. [26] gave a sufficient condition for a quasi-kernel being a kernelled quasidifferential. In [11], Gao presented a condition in terms of Demyanov difference, called g-condition, in which the kernelled quasidifferential exists. The corresponding subclasses and augmented class of g-q.d. functions on $R^{n}$ were defined and some more properties on this class were presented according to Song and Xia [23].

Although the kernelled quasidifferential is known to have good algebraic properties and geometric structure (see [25]), it is still not very convenient for calculating the kernelled quasidifferentials of $-f$ and $\min \left\{f_{i} \mid i \in\right.$ a finite index set $\left.I\right\}$, where $f$ and $f_{i}$ are kernelled quasidifferentiable functions. Hence, in this paper, the notion of adjoint kernelled quasidifferential, which is well-defined for $-f$ and $\min \left\{f_{i} \mid i \in I\right\}$, is employed as a representative of the equivalence class of quasidifferentials. Some algebraic properties of the adjoint kernelled quasidifferential are given and the existence of the adjoint kernelled quasidifferential is explored by means of the minimal quasidifferential and the Demyanov difference of convex sets. The rest of the paper is organized as follows. In Section 2, some preliminary definitions and results used in the paper are provided. In Section 3, definitions of adjoint kernelled quasidifferential will be introduced and some operations of adjoint kernelled quasidifferentiable functions are given. In Section 4, we prove that the adjoint kernelled quasidifferential exists in one- and two-dimensional cases and two sufficient conditions for the existence of the adjoint kernelled quasidifferential in $R^{n}(n \geq 3)$ are given. In Section 5, under some condition, a formula of the adjoint kernelled quasidifferential is presented.

\section{Preliminaries}

The support function $\delta^{*}(\cdot \mid C)$ of a set $C \in Y_{n}$ is defined by

$$
\delta^{*}(x \mid C)=\max _{v \in C}\langle v, x\rangle, \quad \forall x \in R^{n} .
$$

It is well known (see, e.g., [6]) that the mapping $A \mapsto \delta^{*}(\cdot \mid A)$ called the Minkowski duality is one-to-one correspondence between $Y_{n}$ and the set $P_{n}$ of all finite sublinear functions is defined on $R^{n}$.

Proposition 1. Let $A, B \in Y_{n}$; then

$$
A \subset B \Longleftrightarrow \delta^{*}(x \mid A) \leq \delta^{*}(x \mid B), \quad \forall x \in R^{n} .
$$

It is true that $\delta^{*}(\cdot \mid C)$ is convex with

$$
\partial \delta^{*}(x \mid C)=\left\{u \in C \mid\langle u, x\rangle=\max _{v \in C}\langle v, x\rangle\right\},
$$

particularly, $\partial \delta^{*}(0 \mid C)=C$, where $\partial$ denotes the subdifferential in the sense of convex analysis [27].

For any $d \in R^{n}$ and $C \in Y_{n}$, we denote the max-face of $C$ with respect to $d$ by the formula

$$
C(d)=\left\{x \in C \mid\langle d, x\rangle=\delta^{*}(d \mid C)\right\} .
$$

Obviously, the max-face $C(d)$ coincides with the subdifferential $\partial \delta^{*}(d \mid C)$. Denote by $N_{C}(x)$ the normal cone to $C$ at $x \in C$; that is,

$$
N_{C}(x)=\left\{d \in R^{n} \mid\langle d, y-x\rangle \leq 0, \forall y \in C\right\} .
$$

Proposition 2. Let $C \in Y_{n}$, for $x \in C$; it holds

$$
x \in C(d) \Longleftrightarrow d \in N_{C}(x) \text {. }
$$


Proposition 3. Let $C \in Y_{n}$ and $x \in C$. If $d_{1}, d_{2} \in N_{C}(x)$, then

$$
\delta^{*}\left(d_{1}+d_{2} \mid C\right)=\delta^{*}\left(d_{1} \mid C\right)+\delta^{*}\left(d_{2} \mid C\right) .
$$

Let the function $f$ defined on $R^{n}$ be locally Lipschitz continuous and let $D_{f}$ denote the set where $\nabla f$ exists. The Clarke subdifferential $\partial_{\mathrm{Cl}} f(x)$ of $f$ at $x$ is defined as follows:

$$
\partial_{\mathrm{Cl}} f(x)=\operatorname{co}\left\{\lim _{x_{n} \rightarrow x} \nabla f\left(x_{n}\right) \mid x_{n} \longrightarrow x, x_{n} \in D_{f}\right\},
$$

where "co" denotes the convex hull. In the convex case, the Clarke subdifferential coincides with the subdifferential in the sense of convex analysis [28].

A set $T \subset R^{n}$ is called of full measure (with respect to $R^{n}$ ), if $R^{n} \backslash T$ is a set of measure zero. Let $A \in Y_{n}$ and $T_{A}=D_{\delta^{*}(\cdot \mid A)}$ be the set of all points $x \in R^{n}$ such that $\nabla \delta^{*}(x \mid A)$ exists. The set $T_{A}$ is of full measure in $R^{n}$. Let $A, B \in Y_{n}$ and $T$ be a subset of $T_{A} \cap T_{B}$ of full measure; then the set

$$
A \dot{-} B=\operatorname{clco}\left\{\nabla \delta^{*}(x \mid A)-\nabla \delta^{*}(x \mid B) \mid x \in T\right\}
$$

is called Demyanov difference of $A$ and $B$, where "cl" refers to the closure. This construction was applied implicitly by Demyanov for the study of connections between the Clarke subdifferential and the quasidifferential [3]. In general, the Demyanov difference is smaller than the Minkowski difference. It is true that

$$
A \dot{-} B \subset A-B
$$

According to [6], the Demyanov difference can be rewritten as

$$
A \dot{-} B=\left.\partial_{\mathrm{Cl}}\left(\delta^{*}(y \mid A)-\delta^{*}(y \mid B)\right)\right|_{y=0} .
$$

Define the algebraic operations of addition and multiplication by a real number in $Y_{n}^{2}=Y_{n} \times Y_{n}$ and the equivalence relation as follows:

$$
\begin{gathered}
(A, B)+(C, D)=(A+C, B+D), \\
c(A, B)=(c A, c B), \quad c \geq 0, \\
c(A, B)=(c B, c A), \quad c<0, \\
(A, B) \sim(C, D) \Longleftrightarrow A-D=C-B,
\end{gathered}
$$

where $c \in R,(A, B)$, and $(C, D) \in Y_{n}^{2}$. It is easy to check that $\mathscr{D} f(x) \in Y_{n}^{2} / \sim$.

Proposition 4. If $\left[\underline{\partial}_{1} f(x), \bar{\partial}_{1} f(x)\right],\left[\underline{\partial}_{2} f(x), \bar{\partial}_{2} f(x)\right] \quad \epsilon$ D $f(x)$, then

$$
\underline{\partial}_{1} f(x) \dot{-}\left(-\bar{\partial}_{1} f(x)\right)=\underline{\partial}_{2} f(x) \dot{-}\left(-\bar{\partial}_{2} f(x)\right) .
$$

The main formulas of quasidifferential calculus will be stated as Proposition 5. Algebraic operations over quasidifferentials are performed as over elements of the space of compact sets (or what is the same, as over pairs of sets).

Proposition 5. Let $\Delta_{n}(x)$ denote the set of all functions defined on an open set $\mathcal{O} \subset R^{n}$ and quasidifferentiable at a point $x \in \mathcal{O}$. Then, the following hold.
(1) If $f_{1}, f_{2} \in \Delta_{n}(x), c_{1}, c_{2}$ are real numbers, then $c_{1} f_{1}+$ $c_{2} f_{2} \in \Delta_{n}(x)$, and

$$
D\left(c_{1} f_{1}+c_{2} f_{2}\right)(x)=c_{1} D f_{1}(x)+c_{2} D f_{2}(x) .
$$

Note that, in particular, $D(-f(x))=-D f(x)$.

(2) Let $f_{1}, f_{2} \in \Delta_{n}(x)$. Then, $f_{1} \cdot f_{2} \in \Delta_{n}(x)$ and

$D\left(f_{1} \cdot f_{2}\right)(x)=f_{1}(x) D f_{2}(x)+f_{2}(x) D f_{1}(x)$.

(3) If $f \in \Delta_{n}(x), f(x) \neq 0$, then $1 / f$ is quasidifferentiable at $x$ and

$$
D f^{-1}(x)=-f^{-2}(x) D f(x) .
$$

(4) Let $f_{1}, f_{2}, \ldots, f_{n} \in \Delta_{n}(x)$ and

$$
\begin{array}{ll}
g(y)=\max \left(f_{1}(y), \ldots, f_{n}(y)\right), & \forall y \in \mathcal{O}, \\
h(y)=\min \left(f_{1}(y), \ldots, f_{n}(y)\right), & \forall y \in \mathcal{O} .
\end{array}
$$

Then, $g \in \Delta_{n}(x), h \in \Delta_{n}(x)$, and

$$
D g(x)=[\underline{\partial} g(x), \bar{\partial} g(x)], \quad D h(x)=[\underline{\partial} h(x), \bar{\partial} h(x)],
$$

where

$$
\begin{aligned}
& \underline{\partial} g(x)=\text { co } \bigcup_{k \in R(x)}\left(\underline{\partial} f_{k}(x)-\sum_{i \in R(x), i \neq k} \bar{\partial} f_{i}(x)\right) \\
& \bar{\partial} g(x)=\sum_{k \in R(x)} \bar{\partial} f_{k}(x), \quad \underline{\partial} h(x)=\sum_{k \in S(x)} \underline{\partial} f_{k}(x), \quad(22) \\
& \bar{\partial} h(x)=\text { co } \bigcup_{k \in S(x)}\left(\bar{\partial} f_{k}(x)-\sum_{i \in S(x), i \neq k} \underline{\partial} f_{i}(x)\right) . \\
& \text { Here, } R(x)=\left\{i \mid f_{i}(x)=g(x)\right\}, S(x)=\left\{i \mid f_{i}(x)=\right. \\
& h(x)\} .
\end{aligned}
$$

\section{Adjoint Kernelled Quasidifferential}

The kernelled quasidifferential is known to have good algebraic properties (see [25]), but it is still not very convenient for calculating the kernelled quasidifferentials of $-f$ and $\min \left\{f_{i} \mid i \in\right.$ a finite index set $\left.I\right\}$, where $f$ and $f_{i}$ are kernelled quasidifferentiable functions. So it is natural and necessary to explore the pair of sets $[\underline{S}, S]$, where $S$ is defined as in (3) and

$$
\underline{S}=\bigcap_{D f(x) \in \mathscr{D} f(x)}(\underline{\partial} f(x)-\underline{\partial} f(x)) .
$$

Obviously, $\underline{S}$ is nonempty and symmetric. Since having the similar structure to the quasi-kernel of $\mathscr{D} f(x),[\underline{S}, S]$ is called an adjoint quasi-kernel of $\mathscr{D} f(x)$, where $\underline{S}$ and $S$ are called adjoint sub-kernel and adjoint super-kernel, respectively. Of course $\underline{S}$ and $S$ are compact convex. This motivates the introduction of the following notions. 
Definition 6. Let $f \in \Delta_{n}(x)$. The adjoint quasi-kernel is said to be an adjoint kernelled quasidifferential of $f$ at $x$ if and only if

$$
[\underline{S}, S] \in \mathscr{D} f(x) \text {. }
$$

If $f$ has an adjoint kernelled quasidifferential at $x \in R^{n}$, then $f$ is said to be an adjoint kernelled quasidifferentiable function at $x$. The adjoint kernel $[\underline{S}, S]$ is a quasidifferential, denoted by $D_{k^{*}} f(x)=\left[\underline{\partial}_{k^{*}} f(x), \bar{\partial}_{k^{*}} f(x)\right]$.

From the definition of quasidifferential and Proposition 5, the following proposition can be obtained immediately, which is especially useful in the study of the operation rules of adjoint kernelled quasidifferential.

Proposition 7. (1) If $f_{1}, f_{2} \in \Delta_{n}(x), c_{1}, c_{2} \in R$, then

$$
\mathscr{D}\left(c_{1} f_{1}+c_{2} f_{2}\right)(x)=c_{1} \mathscr{D} f_{1}(x)+c_{2} \mathscr{D} f_{2}(x) \text {. }
$$

Note that, in particular, $\mathscr{D}(-f(x))=-\mathscr{D} f(x)$.

(2) Let $f_{1}, f_{2} \in \Delta_{n}(x)$. Then,

$$
\mathscr{D}\left(f_{1} \cdot f_{2}\right)(x)=f_{1}(x) \mathscr{D} f_{2}(x)+f_{2}(x) \mathscr{D} f_{1}(x) .
$$

(3) If $f \in \Delta_{n}(x), f(x) \neq 0$, then

$$
\mathscr{D} f^{-1}(x)=-f^{-2}(x) \mathscr{D} f(x) .
$$

If the adjoint kernelled quasidifferential exists, some operation rules of adjoint kernelled quasidifferential are presented as follows.

Theorem 8. Let $\Delta_{n, k^{*}}(x)$ denote the set of all functions in $\Delta_{n}(x)$ and having adjoint kernelled quasidifferential at $x$. Then, the following hold.

(1) If $f_{1}, f_{2} \in \Delta_{n, k^{*}}(x)$, then $f_{1}+f_{2} \in \Delta_{n, k^{*}}(x)$ and

$$
D_{k^{*}}\left(f_{1}+f_{2}\right)(x)=D_{k^{*}} f_{1}(x)+D_{k^{*}} f_{2}(x) .
$$

(2) If $f,-f \in \Delta_{n, k^{*}}(x), c \in R$, then $c f \in \Delta_{n, k^{*}}(x)$ and

$$
D_{k^{*}} c f(x)=|c| D_{k^{*}}(\operatorname{sign} c) f(x) .
$$

(3) If $f_{1}, f_{2},-f_{1},-f_{2} \in \Delta_{n, k^{*}}(x)$, then $f_{1} \cdot f_{2} \in \Delta_{n, k^{*}}(x)$ and

$$
\begin{aligned}
D_{k^{*}}\left(f_{1} \cdot f_{2}\right)(x)= & \left|f_{1}(x)\right| D_{k^{*}}\left(\operatorname{sign} f_{1}(x)\right) f_{2}(x) \\
& +\left|f_{2}(x)\right| D_{k^{*}}\left(\operatorname{sign} f_{2}(x)\right) f_{1}(x) .
\end{aligned}
$$

(4) If $f,-f \in \Delta_{n, k^{*}}(x), f(x) \neq 0$, then $1 / f \in \Delta_{n, k^{*}}(x)$ and

$$
D_{k^{*}} f^{-1}(x)=f^{-2}(x) D_{k^{*}}(-f(x)) .
$$

Proof. We will prove only Properties (1) and (2). Properties (3) and (4) can be proved in an analogous manner.
(1) Since $f_{1}, f_{2} \in \Delta_{n, k^{*}}(x)$, then

$$
\begin{gathered}
\bigcap_{D f_{1}(x) \in \mathscr{D} f_{1}(x)}\left(\underline{\partial} f_{1}(x)-\underline{\partial} f_{1}(x)\right)=\underline{\partial}_{k^{*}} f_{1}(x), \\
\bigcap_{D f_{2}(x) \in \mathscr{D} f_{2}(x)}\left(\underline{\partial} f_{2}(x)-\underline{\partial} f_{2}(x)\right)=\underline{\partial}_{k^{*}} f_{2}(x) .
\end{gathered}
$$

From Propositions 5 and 7 and (32), it follows that

$$
\begin{gathered}
\bigcap_{D\left(f_{1}+f_{2}\right)(x) \in \mathscr{D}\left(f_{1}+f_{2}\right)(x)}\left(\underline{\partial}\left(f_{1}+f_{2}\right)(x)-\underline{\partial}\left(f_{1}+f_{2}\right)(x)\right) \\
=\bigcap_{D f_{1}(x)+D f_{2}(x) \in \mathscr{D} f_{1}(x)+\mathscr{D} f_{2}(x)}\left(\underline{\partial} f_{1}(x)-\underline{\partial} f_{1}(x)\right. \\
\left.+\underline{\partial} f_{2}(x)-\underline{\partial} f_{2}(x)\right) \\
=\bigcap_{D f_{1}(x) \in \mathscr{D} f_{1}(x)}\left(\underline{\partial} f_{1}(x)-\underline{\partial} f_{1}(x)\right) \\
+\bigcap_{D f_{2}(x) \in \mathscr{D} f_{2}(x)}\left(\underline{\partial} f_{2}(x)-\underline{\partial} f_{2}(x)\right) \\
=\underline{\partial}_{k^{*}} f_{1}(x)+\underline{\partial}_{k^{*}} f_{2}(x) .
\end{gathered}
$$

By the similar way, we can prove that

$$
\begin{gathered}
\bigcap_{D\left(f_{1}+f_{2}\right)(x) \in \mathscr{D}\left(f_{1}+f_{2}\right)(x)}\left(\underline{\partial}\left(f_{1}+f_{2}\right)(x)+\bar{\partial}\left(f_{1}+f_{2}\right)(x)\right) \\
=\bar{\partial}_{k^{*}} f_{1}(x)+\bar{\partial}_{k^{*}} f_{2}(x) .
\end{gathered}
$$

Since $\left[\underline{\partial}_{k^{*}} f_{1}(x)+\underline{\partial}_{k^{*}} f_{2}(x), \bar{\partial}_{k^{*}} f_{1}(x)+\bar{\partial}_{k^{*}} f_{2}(x)\right] \in \mathscr{D} f_{1}(x)+$ $\mathscr{D} f_{2}(x)=\mathscr{D}\left(f_{1}+f_{2}\right)(x)$, hence, together with (33) and (34), one has that $f_{1}+f_{2} \in \Delta_{n, k^{*}}(x)$.

(2) Since $f,-f \in \Delta_{n, k^{*}}(x)$, then, together with Propositions 5 and 7 , one has that

$\bigcap_{D c f(x) \in \mathscr{D} c f(x)}(\underline{\partial} c f(x)-\underline{\partial} c f(x))$

$=\bigcap_{|c| D(\operatorname{sign} c) f(x) \in|c| \mathscr{D}(\operatorname{sign} c) f(x)}|c|(\underline{\partial}(\operatorname{sign} c) f(x)$

$$
-\underline{\partial}(\operatorname{sign} c) f(x))
$$

$$
\begin{aligned}
& =|c| \bigcap_{D(\operatorname{sign} c) f(x) \in \mathscr{D}(\operatorname{sign} c) f(x)}(\underline{\partial} f(x)-\underline{\partial} f(x)) \\
& =|c| \underline{\partial}_{k^{*}}(\operatorname{sign} c) f(x) .
\end{aligned}
$$


Similarly, we can prove that

$$
\bigcap_{D c f(x) \in \mathscr{D} c f(x)}(\underline{\partial} c f(x)+\bar{\partial} c f(x))=|c| \bar{\partial}_{k^{*}}(\operatorname{sign} c) f(x) .
$$

Combining (35) with (36) leads to

$$
\begin{gathered}
{\left[\bigcap_{D c f(x) \in \mathscr{D} c f(x)}(\underline{\partial} c f(x)-\underline{\partial} c f(x)),\right.} \\
\left.\quad \bigcap_{D c f(x) \in \mathscr{D} c f(x)}(\underline{\partial} c f(x)+\bar{\partial} c f(x))\right] \\
\in|c| \mathscr{D}(\operatorname{sign} c) f(x)=\mathscr{D} c f(x) .
\end{gathered}
$$

Hence, $c f \in \Delta_{n, k^{*}}(x)$.

By $\Delta_{n, k}(x)$ we denote the set of all functions in $\Delta_{n}(x)$ and having kernelled quasidifferential at $x$. Obviously, one has that $\Delta_{n, k}(x) \subset \Delta_{n}(x)$. The adjoint kernelled quasidifferential is convenient for calculating $D_{k^{*}} \min \left\{f_{i} \mid i \in I\right\}$ and can calculate the adjoint kernelled quasidifferential of $-f$ with kernelled quasidifferential, where $f, f_{i} \in \Delta_{n, k}(x), i \in$ a finite index set $I$.

Theorem 9. If $f \in \Delta_{n, k}(x)$, then $-f \in \Delta_{n, k^{*}}(x)$ and

$$
D_{k^{*}}(-f)=-D_{k} f(x) \text {. }
$$

If $f \in \Delta_{n, k^{*}}(x)$, then $-f \in \Delta_{n, k}(x)$ and

$$
D_{k}(-f)=-D_{k^{*}} f(x)
$$

Proof. Since $f \in \Delta_{n, k}(x)$, then $D_{k} f(x)=\left[\underline{\partial}_{k} f(x), \bar{\partial}_{k} f(x)\right] \epsilon$ $\mathscr{D} f(x)$, where

$$
\begin{aligned}
& \underline{\partial}_{k} f(x)=\bigcap_{D f(x) \in \mathscr{D} f(x)}(\underline{\partial f}(x)+\bar{\partial} f(x)), \\
& \bar{\partial}_{k} f(x)=\bigcap_{D f(x) \in \mathscr{D} f(x)}(\bar{\partial} f(x)-\bar{\partial} f(x)) .
\end{aligned}
$$

By Propositions 5 and 7 and (41), we obtain

$$
\begin{aligned}
& \bigcap_{D(-f)(x) \in \mathscr{D}(-f)(x)} \underline{\partial}(-f)(x)-\underline{\partial}(-f)(x) \\
& =\bigcap_{D f(x) \in \mathscr{D} f(x)}-(\bar{\partial} f(x)-\bar{\partial} f(x))=-\bar{\partial}_{k} f(x) .
\end{aligned}
$$

From Propositions 5 and 7 and (40), it follows that

$$
\begin{gathered}
\bigcap_{D(-f)(x) \in \mathscr{D}(-f)(x)} \underline{\partial}(-f)(x)+\bar{\partial}(-f)(x) \\
=\bigcap_{D f(x) \in \mathscr{D} f(x)}-(\underline{\partial} f(x)+\bar{\partial} f(x))=-\underline{\partial}_{k} f(x) .
\end{gathered}
$$

Obviously, $\left[-\bar{\partial}_{k} f(x),-\underline{\partial}_{k} f(x)\right]=-D_{k} f(x) \in-\mathscr{D} f(x)=$ $\mathscr{D}(-f)(x)$. This fact, together with (42) and (43), implies that

$$
\begin{gathered}
{\left[\bigcap_{D(-f)(x) \in \mathscr{D}(-f)(x)} \underline{\partial}(-f)(x)-\underline{\partial}(-f)(x),\right.} \\
\left.\bigcap_{D(-f)(x) \in \mathscr{D}(-f)(x)} \underline{\partial}(-f)(x)+\bar{\partial}(-f)(x)\right] \\
\in \mathscr{D}(-f)(x) .
\end{gathered}
$$

Then, $-f \in \Delta_{n, k^{*}}(x)$ and $D_{k^{*}}(-f)(x)=-D_{k} f(x)$. Similarly, it can be proved that if $f \in \Delta_{n, k^{*}}(x)$, then $-f \in \Delta_{n, k}(x)$ and $D_{k}(-f)=-D_{k^{*}} f(x)$. The proof is completed.

Theorem 10. Let $f_{1}, f_{2}, \ldots, f_{n} \in \Delta_{n, k^{*}}(x)$ and

$$
f(y)=\min \left(f_{1}(y), \ldots, f_{n}(y)\right), \quad \forall y \in \mathcal{O}
$$

Then, $f \in \Delta_{n, k^{*}}(x)$ and $D_{k^{*}} f(x)=\left[\underline{\partial}_{k^{*}} f(x), \bar{\partial}_{k^{*}} f(x)\right]$, where

$$
\underline{\partial}_{k^{*}} f(x)=\sum_{i \in S(x)} \underline{\partial}_{k^{*}} f_{i}(x)
$$

$$
\bar{\partial}_{k^{*}} f(x)=\operatorname{co} \bigcup_{i \in S(x)}\left(\bar{\partial}_{k^{*}} f_{i}(x)-\sum_{j \in S(x), j \neq i} \underline{\partial}_{k^{*}} f_{j}(x)\right) \text {. }
$$

Here, $S(x)=\left\{i \mid f_{i}(x)=f(x)\right\}$.

Proof. Since $f_{1}, f_{2}, \ldots, f_{n} \in \Delta_{n, k^{*}}(x)$ and $f(y)=\min \left(f_{1}(y)\right.$, $\left.\ldots, f_{n}(y)\right), \forall y \in \mathcal{O}$, then, according to Propositions 5 and 7 , we have

$$
\begin{aligned}
& \bigcap_{D f(x) \in \mathscr{D} f(x)}(\underline{\partial} f(x)-\underline{\partial} f(x)) \\
& =\bigcap_{D f(x) \in \mathscr{D} f(x)}\left(\sum_{i \in S(x)} \underline{\partial} f_{i}(x)-\sum_{i \in S(x)} \underline{\partial} f_{i}(x)\right) \\
& =\sum_{i \in S(x)} \bigcap_{D f_{i}(x) \in \mathscr{D} f_{i}(x)}\left(\underline{\partial} f_{i}(x)-\underline{\partial} f_{i}(x)\right) \\
& =\sum_{i \in S(x)} \underline{\partial}_{k^{*}} f_{i}(x) .
\end{aligned}
$$

Since, for $C_{i} \in Y_{n}, i \in I$, where $I$ denotes a finite index set, one has that

$$
\operatorname{co\bigcup } \bigcup_{i \in I} C_{i} \bigcup_{\lambda_{i} \geq 0, \sum_{i \in I} \lambda_{i}=1} \sum_{i \in I} \lambda_{i} C_{i}
$$


where $\lambda_{i} \in R, i \in I$. Hence, together with Proposition 5, it follows that

$$
\begin{aligned}
& \underline{\partial} f(x)+\bar{\partial} f(x) \\
& =\sum_{i \in S(x)} \underline{\partial} f_{i}(x)+\operatorname{co} \bigcup_{i \in S(x)}\left(\bar{\partial} f_{i}(x)-\sum_{j \in S(x), j \neq i} \underline{\partial} f_{j}(x)\right) \\
& =\sum_{i \in S(x)} \partial f_{i}(x) \\
& +\bigcup_{\lambda_{i} \geq 0, \sum_{i \in S(x)}}\left(\sum_{i=1} \lambda_{i}\left(\bar{\partial} f_{i}(x)-\sum_{\substack{j \in S(x) \\
j \neq i}} \underline{\partial} f_{j}(x)\right)\right) \\
& \bigcap_{D f(x) \in \mathscr{D} f(x)}(\underline{\partial} f(x)+\bar{\partial} f(x)) \\
& =\bigcap_{D f(x) \in \mathscr{D} f(x)} \bigcup_{\lambda_{i} \geq 0, \sum_{i \in S(x)}} \sum_{\lambda_{i}=1} \lambda_{i}\left(\underline{\partial} f_{i}(x)+\bar{\partial} f_{i}(x)-\sum_{\substack{j \in S(x) \\
j \neq i}}\left(\underline{\partial} f_{j}(x)-\underline{\partial} f_{j}(x)\right)\right) \\
& =\bigcup_{\lambda_{i} \geq 0, \sum_{i \in S(x)}} \sum_{\lambda_{i}=1} \lambda_{i}\left(\bigcap_{i \in S(x)}\left(\underline{\partial} f_{i}(x)+\bar{\partial} f_{i}(x)\right)-\sum_{\substack{j \in S(x) \\
j \neq i}} \bigcap_{\substack{D f_{j} \\
j}} \bigcap_{\substack{i \\
j} \in f_{j}(x)}\left(\underline{\partial} f_{j}(x)-\underline{\partial} f_{j}(x)\right)\right) \\
& =\operatorname{co} \bigcup_{i \in S(x)}\left(\bar{\partial}_{k^{*}} f_{i}(x)-\sum_{j \in S(x), j \neq i} \underline{\partial}_{k^{*}} f_{j}(x)\right) \text {. }
\end{aligned}
$$

$$
\begin{array}{r}
=\bigcup_{\lambda_{i} \geq 0, \sum_{i \in S(x)} \lambda_{i}=1} \sum_{i \in S(x)} \lambda_{i}\left(\underline{\partial} f_{i}(x)+\bar{\partial} f_{i}(x)\right. \\
\left.-\sum_{\substack{j \in S(x) \\
j \neq i}}\left(\underline{\partial} f_{j}(x)-\underline{\partial} f_{j}(x)\right)\right) .
\end{array}
$$

Based on Propositions 5 and 7 and (47) and (50), one has that

$$
\begin{aligned}
& {\left[\bigcap_{D f(x) \in \mathscr{D} f(x)}(\underline{\partial} f(x)-\underline{\partial} f(x)),\right.} \\
& \left.\bigcap_{D f(x) \in \mathscr{D} f(x)}(\underline{\partial} f(x)+\bar{\partial} f(x))\right] \in \mathscr{D} f(x) ;
\end{aligned}
$$

hence $f \in \Delta_{n, k^{*}}(x)$. The demonstration is completed.

\section{Existence of the Adjoint Kernelled Quasidifferential}

In this section, the existence of the adjoint kernelled quasidifferential of a quasidifferentiable function is established. In one- and two-dimensional cases, we prove that the adjoint kernelled quasidifferential exists and give its expression by using of a minimal quasidifferential. We also develop the existence of the adjoint kernelled quasidifferential for a quasidifferentiable function on $R^{n}(n \geq 3)$ under some conditions.
Theorem 11. Suppose that $f \in \Delta_{n}(x), n=1,2$, and $\left[\underline{\partial}_{0}^{m} f(x), \bar{\partial}_{0}^{m} f(x)\right]$ is a minimal quasidifferential of $f$ at $x$. Then, the relations below hold

$$
\underline{S}=\underline{\partial}_{0}^{m} f(x)-\underline{\partial}_{0}^{m} f(x), \quad S=\underline{\partial}_{0}^{m} f(x)+\bar{\partial}_{0}^{m} f(x) .
$$

Furthermore, $f \in \Delta_{n, k^{*}}(x)$; that is, $[\underline{S}, S] \in \mathscr{D} f(x)$.

Proof. Let $[\partial f(x), \bar{\partial} f(x)] \in \mathscr{D} f(x)$. From the existence of the minimal quasidifferentials, see [18], it follows that there exists a minimal quasidifferential of $f$ at $x$, denoted by $\left[\underline{\partial}^{m} f(x), \bar{\partial}^{m} f(x)\right]$, such that $\underline{\partial}^{m} f(x) \subset \underline{\partial} f(x), \bar{\partial}^{m} f(x) \subset$ $\bar{\partial} f(x)$. Consequently,

$$
\begin{aligned}
& \underline{\partial}^{m} f(x)-\underline{\partial}^{m} f(x) \subset \underline{\partial} f(x)-\underline{\partial} f(x), \\
& \underline{\partial}^{m} f(x)+\bar{\partial}^{m} f(x) \subset \underline{\partial} f(x)+\bar{\partial} f(x) .
\end{aligned}
$$

Note that both $\left[\underline{\partial}^{m} f(x), \bar{\partial}^{m} f(x)\right]$ and $\left[\underline{\partial}_{0}^{m} f(x), \bar{\partial}_{0}^{m} f(x)\right]$ are the minimal quasidifferentials of $f$ at $x$. According to the translation property of the equivalent minimal quasidifferentials in the one- and two-dimensional case, see $[15,18]$, there exists $c \in R^{n}, n=1,2$, such that the minimal quasidifferential $\left[\underline{\partial}^{m} f(x), \bar{\partial}^{m} f(x)\right]$ can be expressed as

$$
\left[\underline{\partial}^{m} f(x), \bar{\partial}^{m} f(x)\right]=\left[\underline{\partial}_{0}^{m} f(x)+\{c\}, \bar{\partial}_{0}^{m} f(x)-\{c\}\right] .
$$


This leads to

$$
\begin{aligned}
& \underline{\partial}^{m} f(x)-\underline{\partial}^{m} f(x)=\underline{\partial}_{0}^{m} f(x)-\underline{\partial}_{0}^{m} f(x), \\
& \underline{\partial}^{m} f(x)+\bar{\partial}^{m} f(x)=\underline{\partial}_{0}^{m} f(x)+\bar{\partial}_{0}^{m} f(x) .
\end{aligned}
$$

It follows from (53a), (53b), (55a), and (55b) that

$$
\begin{aligned}
& \underline{\partial}_{0}^{m} f(x)-\underline{\partial}_{0}^{m} f(x) \subset \underline{\partial} f(x)-\underline{\partial} f(x), \\
& \underline{\partial}_{0}^{m} f(x)+\bar{\partial}_{0}^{m} f(x) \subset \underline{\partial f}(x)+\bar{\partial} f(x) .
\end{aligned}
$$

Taking the intersection on the right hands of (56a) and of (56b) for all quasidifferentials of $f$ at $x$, we have that

$$
\begin{aligned}
& \underline{\partial}_{0}^{m} f(x)-\underline{\partial}_{0}^{m} f(x) \subset \underline{S}, \\
& \underline{\partial}_{0}^{m} f(x)+\bar{\partial}_{0}^{m} f(x) \subset S .
\end{aligned}
$$

On the other hand, $\left[\underline{\partial}_{0}^{m} f(x), \bar{\partial}_{0}^{m} f(x)\right] \in \mathscr{D} f(x)$ implies that

$$
\begin{aligned}
& \underline{S} \subset \underline{\partial}_{0}^{m} f(x)-\underline{\partial}_{0}^{m} f(x), \\
& S \subset \underline{\partial}_{0}^{m} f(x)+\bar{\partial}_{0}^{m} f(x) .
\end{aligned}
$$

The relations (57a), (57b), (58a), and (58b) lead to that

$$
\begin{gathered}
\underline{S}=\underline{\partial}_{0}^{m} f(x)-\underline{\partial}_{0}^{m} f(x), \\
S=\underline{\partial}_{0}^{m} f(x)+\bar{\partial}_{0}^{m} f(x) .
\end{gathered}
$$

Note that $\left[\underline{\partial}_{0}^{m} f(x), \bar{\partial}_{0}^{m} f(x)\right] \in \mathscr{D} f(x)$ and $\underline{\partial}_{0}^{m} f(x) \in Y_{n}, n=$ 1,2 . Hence,

$$
\left[\underline{\partial}_{0}^{m} f(x)-\underline{\partial}_{0}^{m} f(x), \underline{\partial}_{0}^{m} f(x)+\bar{\partial}_{0}^{m} f(x)\right] \in \mathscr{D} f(x) .
$$

Equations (59a), (59b), and (60) show that $[\underline{S}, S] \in \mathscr{D} f(x)$. The proof is completed.

The conclusion of Theorem 11 strongly depends upon the translation of minimal quasidifferentials. Unfortunately, the minimal quasidifferential is not uniquely determined up to a translation in $R^{n}$ if $n \geq 3$ [15]. But. by the tool of Demyanov difference of compact convex sets, we get the following interesting result about minimal quasidifferential.

Proposition 12. Suppose that $f \in \Delta_{n}(x)$ and there exists a quasidifferential $\left[\underline{\partial}_{0} f(x), \bar{\partial}_{0} f(x)\right] \in \mathscr{D} f(x)$ such that

$$
\underline{\partial}_{0} f(x) \dot{-}\left(-\bar{\partial}_{0} f(x)\right)=\underline{\partial}_{0} f(x)-\left(-\bar{\partial}_{0} f(x)\right) \text {. }
$$

Then $\left[\underline{\partial}_{0} f(x), \bar{\partial}_{0} f(x)\right]$ is a minimal quasidifferential of $f$ at $x$.

Proof. Let $[\underline{\partial} f(x), \bar{\partial} f(x)] \in \mathscr{D} f(x)$ and

$$
\underline{\partial f}(x) \subset \underline{\partial}_{0} f(x), \quad \bar{\partial} f(x) \subset \bar{\partial}_{0} f(x) .
$$

Obviously, one has

$$
\underline{\partial} f(x)+\bar{\partial} f(x) \subset \underline{\partial}_{0} f(x)+\bar{\partial}_{0} f(x) .
$$

By Proposition 4 and (61), we obtain

$$
\begin{aligned}
\underline{\partial}_{0} f(x)+\bar{\partial}_{0} f(x) & =\underline{\partial}_{0} f(x) \dot{-}\left(-\bar{\partial}_{0} f(x)\right) \\
& =\underline{\partial} f(x) \dot{-}(-\bar{\partial} f(x)) .
\end{aligned}
$$

From (13) and (64), it follows that

$$
\underline{\partial}_{0} f(x)+\bar{\partial}_{0} f(x) \subset \underline{\partial} f(x)+\bar{\partial} f(x) .
$$

Combining (63) with (65) leads to

$$
\underline{\partial}_{0} f(x)+\bar{\partial}_{0} f(x)=\underline{\partial} f(x)+\bar{\partial} f(x) .
$$

According to (62) and (66), we conclude that

$$
\underline{\partial} f(x)=\underline{\partial}_{0} f(x), \quad \bar{\partial} f(x)=\bar{\partial}_{0} f(x) .
$$

Then, by the definition of the minimal quasidifferential, $\left[\underline{\partial}_{0} f(x), \bar{\partial}_{0} f(x)\right]$ is a minimal quasidifferential of $f$ at $x$.

Inspired by Proposition 12, we present the following theorem, which gives a sufficient condition for the existence of the adjoint kernelled quasidifferential in $R^{n}(n \geq 3)$.

Theorem 13. Suppose that $f \in \Delta_{n}(x)$ and there exists a quasidifferential $\left[\underline{\partial}_{0} f(x), \bar{\partial}_{0} f(x)\right] \in \mathscr{D} f(x)$ such that

$$
\underline{\partial}_{0} f(x) \dot{-}\left(-\bar{\partial}_{0} f(x)\right)=\underline{\partial}_{0} f(x)-\left(-\bar{\partial}_{0} f(x)\right) .
$$

Then, one has

$$
\begin{aligned}
& \underline{S}=\underline{\partial}_{0} f(x)-\underline{\partial}_{0} f(x), \\
& S=\underline{\partial}_{0} f(x)+\bar{\partial}_{0} f(x) .
\end{aligned}
$$

Furthermore, $[\underline{S}, S] \in \mathscr{D} f(x)$; that is, $f \in \Delta_{n, k^{*}}(x)$.

Proof. Let $[\underline{\partial} f(x), \bar{\partial} f(x)] \in \mathscr{D} f(x)$. From Proposition 4 and (68), it follows that

$$
\begin{aligned}
\underline{\partial}_{0} f(x)+\bar{\partial}_{0} f(x) & =\underline{\partial}_{0} f(x) \dot{-}\left(-\bar{\partial}_{0} f(x)\right) \\
& =\underline{\partial} f(x) \dot{-}(-\bar{\partial} f(x)) \subset \underline{\partial} f(x)+\bar{\partial} f(x) .
\end{aligned}
$$

By the definition of the quasidifferential, it is easy to check that $[U, V] \in \mathscr{D} f(x)$ implies $[U-U, U+V] \in \mathscr{D} f(x)$. Therefore, we have $[\underline{\partial} f(x)-\underline{\partial} f(x), \underline{\partial} f(x)+\bar{\partial} f(x)] \in \mathscr{D} f(x)$ and $\left[\underline{\partial}_{0} f(x)-\underline{\partial}_{0} f(x), \underline{\partial}_{0} f(x)+\bar{\partial}_{0} f(x)\right] \in \mathscr{D} f(x)$. These give

$$
\begin{aligned}
\delta^{*}(y \mid \underline{\partial} f(x)-\underline{\partial} f(x))-\delta^{*}(y \mid-(\underline{\partial} f(x)+\bar{\partial} f(x))) \\
=\delta^{*}\left(y \mid \underline{\partial}_{0} f(x)-\underline{\partial}_{0} f(x)\right) \\
-\delta^{*}\left(y \mid-\left(\underline{\partial}_{0} f(x)+\bar{\partial}_{0} f(x)\right)\right), \quad \forall y \in R^{n} .
\end{aligned}
$$

By (70) and Proposition 1, we obtain

$$
\delta^{*}\left(y \mid \underline{\partial}_{0} f(x)+\bar{\partial}_{0} f(x)\right) \leq \delta^{*}(y \mid \underline{\partial} f(x)+\bar{\partial} f(x)),
$$

$\forall y \in R^{n}$. 
Evidently, (72) is equivalent to the following:

$$
\begin{aligned}
- & \delta^{*}\left(-y \mid \underline{\partial}_{0} f(x)+\bar{\partial}_{0} f(x)\right) \\
& \geq-\delta^{*}(-y \mid \underline{\partial} f(x)+\bar{\partial} f(x)), \quad \forall y \in R^{n} .
\end{aligned}
$$

Combining (71) with (73) leads to

$$
\begin{array}{r}
\delta^{*}\left(y \mid \underline{\partial}_{0} f(x)-\underline{\partial}_{0} f(x)\right) \leq \delta^{*}(y \mid \underline{\partial} f(x)-\underline{\partial} f(x)), \\
\forall y \in R^{n} .
\end{array}
$$

Based on (74) and Proposition 1, one has that

$$
\underline{\partial}_{0} f(x)-\underline{\partial}_{0} f(x) \subset \underline{\partial} f(x)-\underline{\partial} f(x) .
$$

Notice that both (70) and (75) hold for any $[\underline{\partial} f(x), \bar{\partial} f(x)] \epsilon$ $\mathscr{D} f(x)$. Taking the intersection on the right-hand sides of (70) and of (75), respectively, for all quasidifferentials of $f$ at $x$, it is obtained that

$$
\begin{aligned}
& \underline{\partial}_{0} f(x)-\underline{\partial}_{0} f(x) \subset \underline{S}, \\
& \underline{\partial}_{0} f(x)+\bar{\partial}_{0} f(x) \subset S .
\end{aligned}
$$

On the other hand, $\left[\underline{\partial}_{0} f(x), \bar{\partial}_{0} f(x)\right] \in \mathscr{D} f(x)$ implies

$$
\begin{aligned}
& \underline{S} \subset \underline{\partial}_{0} f(x)-\underline{\partial}_{0} f(x), \\
& S \subset \underline{\partial}_{0} f(x)+\bar{\partial}_{0} f(x) .
\end{aligned}
$$

Combining (76a) with (77b) yields (69a). Likewise, (76b) and (77b) yield (69b). Notice that the relation $\left[\underline{\partial}_{0} f(x)-\right.$ $\left.\underline{\partial}_{0} f(x), \underline{\partial}_{0} f(x)+\bar{\partial}_{0} f(x)\right] \in \mathscr{D} f(x)$ has been claimed. We thus complete the proof of the theorem.

A decomposition structure of $f^{\prime}(x ; \cdot)$ is defined by

$$
f^{\prime}(x ; \cdot)=\underline{f}^{\prime}(x ; \cdot)-\bar{f}^{\prime}(x ; \cdot),
$$

where $f^{\prime}(x ; \cdot)$ and $\bar{f}^{\prime}(x ; \cdot)$ are defined by

$$
\begin{gathered}
\underline{f}^{\prime}(x ; \cdot)=\inf _{D f(x) \in \mathscr{D} f(x)} \delta^{*}(\cdot \mid \underline{\partial} f(x)-\underline{\partial} f(x)), \\
\bar{f}^{\prime}(x ; \cdot)=\inf _{D f(x) \in \mathscr{D} f(x)} \delta^{*}(\cdot \mid-(\underline{\partial} f(x)+\bar{\partial} f(x))),
\end{gathered}
$$

respectively. Generally, $f^{\prime}$ and $\bar{f}^{\prime}$ are positively homogeneous, but not sublinear. It is easy to be seen that

$$
\delta^{*}(\cdot \mid \underline{S}) \leq \underline{f}^{\prime}(x ; \cdot), \quad \delta^{*}(\cdot \mid-S) \leq \bar{f}^{\prime}(x ; \cdot) .
$$

It is easy to be seen that, for any $u \in S$, there exists at least one sequence $\left\{u_{i} \mid u_{i} \in \underline{\partial}_{i} f(x)-\underline{\partial}_{i} f(x)\right\}$ convergent to $u$, where $\left[\underline{\partial}_{i} f(x), \bar{\partial}_{i} f(x)\right] \in \mathscr{D} f(x)$. According to Proposition 2, if $u \in \underline{S}$ and $d \in R^{n}$ such that there exist sequences $\left\{u_{i} \mid u_{i} \in\right.$ $\left.\underline{\partial}_{i} f(x)-\underline{\partial}_{i} f(x)\right\}_{i=1}^{\infty} \rightarrow u$ and $\left\{d_{i} \mid d_{i} \in N_{\underline{\partial}_{i} f(x)-\underline{\partial}_{i} f(x)}\left(u_{i}\right)\right\} \rightarrow$ $d$, then $d \in N_{\underline{S}}(u)$ and $\delta^{*}(d \mid \underline{S})=f^{\prime}(x ; d)$.
The above lines enable us to give the following theorem which provides a sufficient condition for $[\underline{S}, S]$ to be an adjoint kernelled quasidifferential.

Let $\mathscr{F}(\underline{S},-S)$ be a shape of $(\underline{S},-S)$ that is defined by a similar way according to [18], such that

$$
\operatorname{clco} \bigcup_{d \in \mathscr{F}(\underline{S},-S)} \underline{S}(d)=\underline{S}, \quad \operatorname{clco} \bigcup_{d \in \mathscr{F}(\underline{S},-S)}-S(d)=-S \text {. }
$$

Theorem 14. Let $f \in \Delta_{n}(x)$ and suppose that $f^{\prime}(x ; \cdot)$ and $\bar{f}^{\prime}(x ; \cdot)$ are continuous with respect to direction, and, furthermore, there exists a shape $\mathscr{F}(\underline{S},-S)$ of $(\underline{S},-S)$ such that, for any $u \in \underline{S}$ and $v \in-S$, one has that

$$
\begin{aligned}
N_{\underline{S}}(u) & =\overline{\text { cone }}\left\{N_{\underline{S}}(u) \cap \mathscr{F}(\underline{S},-S)\right\}, \\
N_{-S}(v) & =\overline{\text { cone }}\left\{N_{-S}(v) \cap \mathscr{F}(\underline{S},-S)\right\},
\end{aligned}
$$

where $\overline{\text { cone }}$ denotes the closed convex conical hull. If, for any $d \in \mathscr{F}(\underline{S},-S), u \in \underline{S}(d)$, and $v \in-S(d)$, there exist sequences

$$
\begin{gathered}
\left\{u_{i} \mid u_{i} \in \underline{\partial}_{i} f(x)-\underline{\partial}_{i} f(x)\right\}_{i=1}^{\infty} \longrightarrow u, \\
\left\{v_{i} \mid v_{i} \in-\left(\underline{\partial}_{i} f(x)+\bar{\partial}_{i} f(x)\right)\right\}_{i=1}^{\infty} \longrightarrow v, \\
\left\{d_{i} \mid d_{i} \in N_{\underline{\partial}_{i} f(x)-\underline{\partial}_{i} f(x)}\left(u_{i}\right) \cap N_{-\left(\underline{\partial}_{i} f(x)+\bar{\partial}_{i} f(x)\right)}\left(v_{i}\right)\right\},
\end{gathered}
$$

such that $d$ is one of clusters of $\left\{d_{i}\right\}_{i=1}^{\infty}$; then $[\underline{S}, S] \in \mathscr{D} f(x)$, that is, $f \in \Delta_{n, k^{*}}(x)$.

Proof. Let $d \in R^{n}$ be an arbitrary nonzero vector. There exist $u \in \underline{S}$ and $v \in-S$ such that $d \in N_{\underline{S}}(u) \cap N_{-S}(v)$. According to (82), there exists a sequence

$$
\begin{aligned}
d_{i} & \in \overline{\text { cone }}\left\{N_{\underline{S}}(u) \cap \mathscr{F}(\underline{S},-S)\right\} \\
& \cap \overline{\text { cone }}\left\{N_{-S}(v) \cap \mathscr{F}(\underline{S},-S)\right\},
\end{aligned}
$$

$i=1,2, \ldots$, convergent to $d$. For each $i$, there are two index sets $J_{i}$ and $\bar{J}_{i}$, with finite indices such that

$$
\begin{gathered}
\underline{d}_{i j} \in N_{\underline{S}}\left(u_{i}\right) \cap \mathscr{F}(\underline{S},-S), \quad j \in \underline{J}_{i}, \\
\bar{d}_{i j} \in N_{-S}\left(v_{i}\right) \cap \mathscr{F}(\underline{S},-S), \quad j \in \bar{J}_{i}, \\
d_{i} \in \operatorname{co}\left\{\underline{d}_{i j} \mid j \in \underline{J}_{i}\right\} \cap \operatorname{co}\left\{\bar{d}_{i j} \mid j \in \bar{J}_{i}\right\} .
\end{gathered}
$$

It follows from (83)-(85) and (87) that, for each $i j$, there exist $\left\{\underline{d}_{i j_{k}}\right\}_{k=1}^{\infty},\left\{\bar{d}_{i j_{k}}\right\}_{k=1}^{\infty},\left\{u_{i j_{k}}\right\}_{k=1}^{\infty}$, and $\left\{v_{i j_{k}}\right\}_{k=1}^{\infty}$ such that

$$
\begin{gathered}
\left\{u_{i j_{k}} \in \underline{\partial}_{i j_{k}} f(x)-\underline{\partial}_{i j_{k}} f(x)\right\}_{k=1}^{\infty} \longrightarrow u_{i}, \\
\left\{v_{i j_{k}} \in-\left(\underline{\partial}_{i j_{k}} f(x)+\bar{\partial}_{i j_{k}} f(x)\right)\right\}_{k=1}^{\infty} \longrightarrow v_{i}, \\
\left\{\underline{d}_{i j_{k}} \in N_{\underline{\partial}_{i j_{k}} f(x)-\underline{\partial}_{i j_{k}} f(x)}\left(u_{i j_{k}}\right)\right\}_{k=1}^{\infty} \longrightarrow \underline{d}_{i j}, \\
j \in \underline{J}_{i}, \quad i=1,2, \ldots, \\
\left\{\bar{d}_{i j_{k}} \in N_{-\left(\underline{\partial}_{i j_{k}} f(x)+\bar{\partial}_{i j_{k}} f(x)\right)}\left(v_{i j_{\mathrm{k}}}\right)\right\}_{k=1}^{\infty} \longrightarrow \bar{d}_{i j}, \\
j \in \bar{J}_{i}, \quad i=1,2, \ldots
\end{gathered}
$$


Since each $d_{i}$ is a convex combination of $\underline{d}_{i j}, j \in \underline{J}_{i}$, or of $\bar{d}_{i j}$, $j \in \bar{J}_{i}$, one has that there are $\underline{\lambda}_{i j} \geq 0$ and $\bar{\lambda}_{i j} \geq 0$ such that

$$
\sum_{j \in \underline{J}_{i}} \underline{\lambda}_{i j}=1, \quad \sum_{j \in \bar{J}_{i}} \bar{\lambda}_{i j}=1
$$

satisfying

$$
\begin{gathered}
d_{i}=\sum_{j \in \underline{J}_{i}} \underline{\lambda}_{i j} \underline{d}_{i j}=\sum_{j \in \bar{J}_{i}} \bar{\lambda}_{i j} \bar{d}_{i j}, \\
\delta^{*}\left(d_{i} \mid \underline{S}\right)=\sum_{j \in \underline{J}_{i}} \underline{\lambda}_{i j}\left\langle\underline{d}_{i j}, u_{i}\right\rangle=\sum_{j \in J_{i}} \underline{\lambda}_{i j_{k}} \lim _{k \rightarrow \infty}\left\langle\underline{d}_{i j_{k}}, u_{i j_{k}}\right\rangle
\end{gathered}
$$

from (83) and (84), where $\underline{d}_{i j_{k}} \in N_{\underline{\partial}_{i j_{k}} f(x)-\underline{\partial}_{i j_{k}} f(x)}\left(u_{i j_{k}}\right)$. Since $\left\{u_{i j_{k}} \in \underline{\partial}_{i j_{k}} f(x)-\underline{\partial}_{i j_{k}} f(x)\right\}_{k=1}^{\infty} \rightarrow u_{i},\left\{\underline{d}_{i j_{k}} \in\right.$ $\left.N_{\underline{\partial}_{j_{k}} f(x)-\underline{\partial}_{i j_{k}} f(x)}\left(u_{i j_{k}}\right)\right\}_{k=1}^{\infty} \rightarrow \underline{d}_{i j}$, it follows, from the sufficient condition for $\delta^{*}(d \mid \underline{S})=\underline{f}^{\prime}(x ; d)$ given before the theorem, that

$$
\delta^{*}\left(\underline{d}_{i j} \mid \underline{s}\right)=\underline{f}^{\prime}\left(x ; \underline{d}_{i j}\right)=\lim _{k \rightarrow \infty}\left\langle\underline{d}_{i j_{k}}, u_{i j_{k}}\right\rangle=\left\langle\underline{d}_{i j}, u_{i}\right\rangle .
$$

Thus, it follows from (91) that

$$
\delta^{*}\left(d_{i} \mid \underline{S}\right)=\left\langle\sum_{j \in \underline{J}_{i}} \underline{\lambda}_{i j} \underline{d}_{i j}, u_{i}\right\rangle=\underline{f}^{\prime}\left(x ; \underline{d}_{i}\right) .
$$

Without loss of generality, assume $\left\{d_{i}\right\}_{i=1}^{\infty} \rightarrow d$. Taking the limit to (92), one has that

$$
\delta^{*}(d \mid \underline{S})=\langle d, u\rangle=\lim _{i \rightarrow \infty} \underline{f}^{\prime}\left(x ; \underline{d}_{i}\right) .
$$

According to the continuity of $\underline{f}^{\prime}(x ; \cdot),(93)$ becomes

$$
\delta^{*}(d \mid \underline{S})=\underline{f}^{\prime}(x ; d) .
$$

Similarly, it can be proved that

$$
\delta^{*}(d \mid-S)=\bar{f}^{\prime}(x ; d) .
$$

According to (94) and (95), we conclude

$$
f^{\prime}(x ; d)=\underline{f}^{\prime}(x ; d)-\bar{f}^{\prime}(x ; d)=\delta^{*}(d \mid \underline{S})-\delta^{*}(d \mid-S) .
$$

Then, by the definition of the quasidifferential, one has $[\underline{S}, S] \in \mathscr{D} f(x)$, that is, $f \in \Delta_{n, k^{*}}(x)$. The demonstration is completed.

\section{Formula of Representative for Quasidifferentials}

Theorem 13 only gives the existence of the adjoint kernelled quasidifferential but does not show us how to calculate it. For the practical purpose, we expect to find a way to calculate a representative of the equivalent class of quasidifferentials for a given quasidifferential. The present section is devoted to this topic.
Lemma 15. Let $[A, B],[U, V] \in \mathscr{D} f(x)$. Then, $U$ has the following form:

$$
U=(A-V) \dot{-}(-B)
$$

Proof. Evidently,

$$
\begin{array}{r}
\delta^{*}(y \mid A)-\delta^{*}(y \mid-B)=\delta^{*}(y \mid U)-\delta^{*}(y \mid-V), \\
\forall y \in R^{n} .
\end{array}
$$

This leads to

$$
\delta^{*}(y \mid A-V)-\delta^{*}(y \mid-B)=\delta^{*}(y \mid U), \quad \forall y \in R^{n} .
$$

Taking the Clarke subdifferential at $y=0$, (99) becomes

$$
\begin{aligned}
\partial_{\mathrm{Cl}} & \left.\left(\delta^{*}(y \mid A-V)-\delta^{*}(y \mid-B)\right)\right|_{y=0} \\
= & \left.\partial_{\mathrm{Cl}}\left(\delta^{*}(y \mid U)\right)\right|_{y=0} .
\end{aligned}
$$

Based on the definition of the Demyanov difference, (100) yields $(A-V) \dot{-}(-B)=U$; that is, (97) holds.

Theorem 16. Let $f \in \Delta_{n}(x)$ and $[\underline{\partial} f(x), \bar{\partial} f(x)] \in \mathscr{D} f(x)$. If there exists $W \in Y_{n}$ such that $[W, \underline{\partial} f(x) \dot{-}(-\bar{\partial} f(x))] \epsilon$ D $f(x)$, then

$$
W=\{\underline{\partial} f(x)-(\underline{\partial} f(x) \dot{-}(-\bar{\partial} f(x)))\} \dot{-}(-\bar{\partial} f(x)) .
$$

Proof. Setting $[A, B]=[\underline{\partial} f(x), \bar{\partial} f(x)]$ and $[U, V]=[W$, $\underline{\partial} f(x) \dot{-}(-\bar{\partial} f(x))]$ in Lemma 15, we have

$$
\begin{aligned}
W= & (A-V) \dot{-}(-B)=\{\underline{\partial} f(x)-(\underline{\partial} f(x) \dot{-}(-\bar{\partial} f(x)))\} \\
& \dot{-}(-\bar{\partial} f(x)) .
\end{aligned}
$$

This completes the proof of the theorem.

Theorem 17. Let $f \in \Delta_{n}(x)$. If there exists $[A, B] \in \mathscr{D} f(x)$ satisfying $A-(-B)=A-(-B)$, then, for any $[\partial f(x), \bar{\partial} f(x)] \epsilon$ $\mathscr{D} f(x)$, the pair of sets

$$
\begin{aligned}
& {[\{\underline{\partial} f(x)-(\underline{\partial} f(x) \dot{-}(-\bar{\partial} f(x)))\}} \\
& \quad \dot{-}(-\bar{\partial} f(x)), \underline{\partial f} f(x) \dot{-}(-\bar{\partial} f(x))]
\end{aligned}
$$

is the adjoint kernelled quasidifferential of $f$.

Proof. By Theorem 13 and Proposition 4,

$$
[A-A, A \dot{-}(-B)]=[A-A, \underline{\partial} f(x) \dot{-}(-\bar{\partial} f(x))]
$$

is the kernelled quasidifferential. According to Theorem 16, $[A-A, \underline{\partial} f(x) \dot{-}(-\bar{\partial} f(x))] \in \mathscr{D} f(x)$ leads to

$$
A-A=\{\underline{\partial} f(x)-(\underline{\partial} f(x) \dot{-}(-\bar{\partial} f(x)))\} \dot{-}(-\bar{\partial} f(x)) .
$$

This means that (103) is the kernelled quasidifferential. The proof is concluded. 
Noticing that the Demyanov difference and the Minkowski difference of polyhedra are polyhedra, we have the following corollary.

Corollary 18. Suppose that there exist $[A, B] \in \mathscr{D} f(x)$ satisfying $A-(-B)=A-(-B)$ and a pair of polyhedra $[U, V] \in \mathscr{D} f(x)$. Then, the kernelled quasidifferential is a pair of polyhedra.

Based on above two theorems, given a quasidifferential, the adjoint kernelled quasidifferential can be formulated under some conditions, for instance, the condition in Theorem 13. In particular, if a polyhedral quasidifferential is given, the adjoint kernelled quasidifferential can be calculated because the Demyanov difference of polyhedra can be calculated (for instance, see [9]).

\section{Conflict of Interests}

The authors declare that there is no conflict of interests regarding the publication of this paper.

\section{Acknowledgment}

This paper is supported by the National Natural Science Foundation of China under Grant no. 11171049.

\section{References}

[1] R. Baier, E. Farkhi, and V. Roshchina, "The directed and Rubinov subdifferentials of quasidifferentiable functions-part I: definition and examples," Nonlinear Analysis: Theory, Methods \& Applications, vol. 75, no. 3, pp. 1074-1088, 2012.

[2] R. Baier, E. Farkhi, and V. Roshchina, "The directed and Rubinov subdifferentials of quasidifferentiable functions-part II: calculus," Nonlinear Analysis: Theory, Methods \& Applications, vol. 75, no. 3, pp. 1058-1073, 2012.

[3] V. F. Demyanov, "On a relation between the Clarke subdifferential and quasidifferential," Vestnik Leningrad University, vol. 13, pp. 183-189, 1981.

[4] V. F. Demyanov and Z. Q. Xia, Minimal Quasidifferentials, International Institute for Applied Systems Analysis (IIASA), Laxenburg, Austria, 1984.

[5] V. F. Demyanov and A. M. Rubinov, Quasidifferential Calculus, Optimization Software, New York, NY, USA, 1986.

[6] V. F. Demyanov and A. M. Rubinov, Constructive Nonsmooth Analysis, vol. 7 of Approximation \& Optimization, Peter Lang, Frankfurt, Germany, 1995.

[7] V. F. Dem'yanov, G. E. Stavroulakis, L. N. Polyakova, and P. D. Panagiotopoulos, Quasidifferentiability and Nonsmooth Modelling in Mechanics, Engineering and Economics, vol. 10 of Nonconvex Optimization and Its Applications, Kluwer Academic, Dordrecht, The Netherlands, 1996.

[8] V. F. Demyanov and A. M. Rubinov, Quasidifferentiability and Related Topics, Kluwer Academic, Dodrecht, The Netherlands, 2000.

[9] Y. Gao, "Demyanov difference of two sets and optimality conditions of Lagrange multiplier type for constrained quasidifferentiable optimization," Journal of Optimization Theory and Applications, vol. 104, no. 2, pp. 377-394, 2000.
[10] Y. Gao, "Representation of the Clarke generalized Jacobian via the quasidifferential," Journal of Optimization Theory and Applications, vol. 123, no. 3, pp. 519-532, 2004.

[11] Y. Gao, "Representative of quasidifferentials and its formula for a quasidifferentiable function," Set-Valued Analysis, vol. 13, no. 4, pp. 323-336, 2005.

[12] Y. Gao, "Calculating the proximal subdifferential via the quasidifferential," Applied Mathematics Letters, vol. 21, no. 11, pp. 11721176, 2008.

[13] B. Luderer and D. Wagner, "Algorithms of quasidifferentiable optimization for the separation of point sets," in Optimization and Optimal Control: Theory and Applications, vol. 39 of Springer Optimization and Its Applications, pp. 157-167, Springer, New York, NY, USA, 2010.

[14] M. R. Deng and Y. Gao, "A property on quasidifferentials," Chinese Journal of Operations Research, vol. 10, no. 1, pp. 65-67, 1991 (Chinese).

[15] J. Grzybowski, "Minimal pairs of convex compact sets," Archiv der Mathematik, vol. 63, no. 2, pp. 173-181, 1994.

[16] Y. Gao, "The star-kernel for a quasidifferentiable function in one dimensional space," Journal of Mathematical Research and Exposition, vol. 8, no. 1, p. 152, 1988.

[17] Y. Gao, Z. Q. Xia, and L. W. Zhang, "Kernelled quasidifferential for a quasidifferentiable function in two-dimensional space," Journal of Convex Analysis, vol. 8, no. 2, pp. 401-408, 2001.

[18] D. Pallaschke, S. Scholtes, and R. Urbański, "On minimal pairs of convex compact sets," Bulletin of the Polish Academy of Sciences. Mathematics, vol. 39, no. 1-2, pp. 105-109, 1991.

[19] D. Pallaschke and R. Urbański, "A continuum of minimal pairs of compact convex sets which are not connected by translations," Journal of Convex Analysis, vol. 3, no. 1, pp. 83-95, 1996.

[20] D. Pallaschke and R. Urbański, "Some criteria for the minimality of pairs of compact convex sets," Zeitschrift für Operations Research, vol. 37, no. 2, pp. 129-150, 1993.

[21] D. Pallaschke and R. Urbański, "Reduction of quasidifferentials and minimal representations," Mathematical Programming, vol. 66, no. 2, pp. 161-180, 1994.

[22] S. Scholtes, "Minimal pairs of convex bodies in two dimensions," Mathematika, vol. 39, no. 2, pp. 267-273, 1992.

[23] C. L. Song and Z. Q. Xia, "A note on the kernelled quasidifferential in the n-dimensional space," Fuzzy Engineering and Operations Research, vol. 147, pp. 541-547, 2012.

[24] Z. Q. Xia, The *-Kernel for Quasidifferntiable Functions, WP-8789, IIASA, Laxenburg, Austria, 1987.

[25] Z. Q. Xia, "On quasidifferential kernels," Demonstratio Mathematica, vol. 26, no. 1, pp. 159-182, 1993.

[26] L. W. Zhang, Z. Q. Xia, Y. Gao, and M. Z. Wang, "Star-kernels and star-differentials in quasidifferential analysis," Journal of Convex Analysis, vol. 9, no. 1, pp. 139-158, 2002.

[27] R. T. Rockafellar, Convex Analysis, Princeton Mathematical Series, Princeton University Press, Princeton, NJ, USA, 1970.

[28] F. H. Clarke, Optimization and Nonsmooth Analysis, Canadian Mathematical Society Series of Monographs and Advanced Texts, Wiley-Interscience, New York, NY, USA, 1983. 


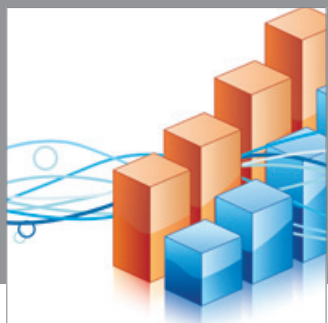

Advances in

Operations Research

mansans

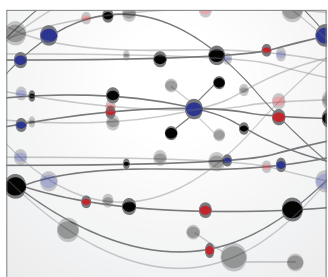

The Scientific World Journal
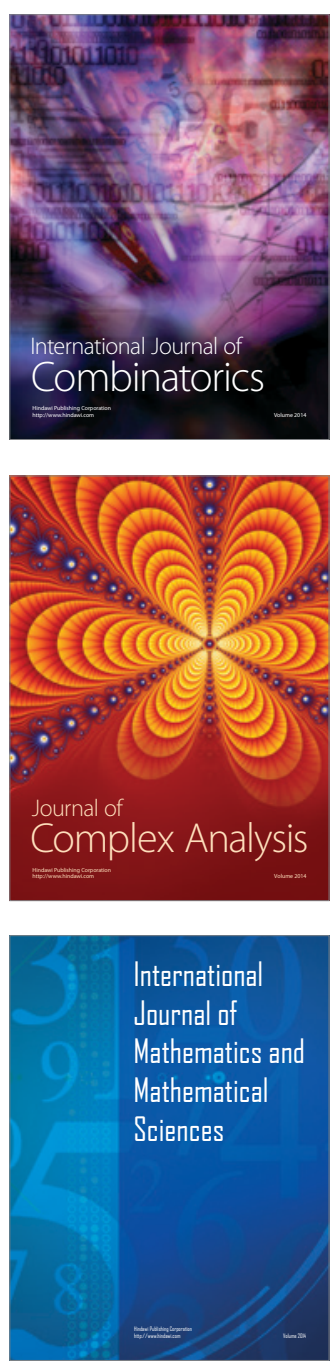
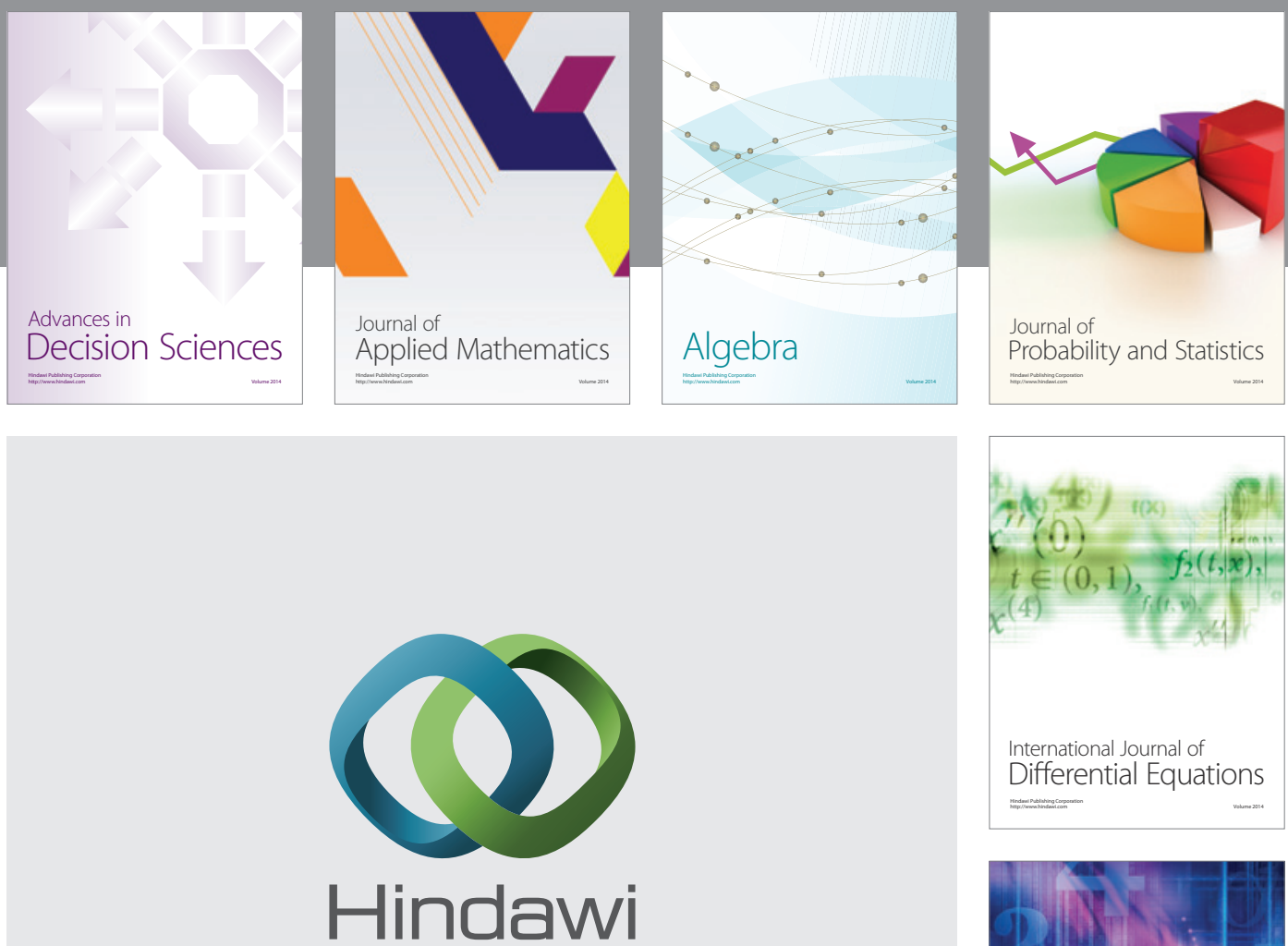

Submit your manuscripts at http://www.hindawi.com
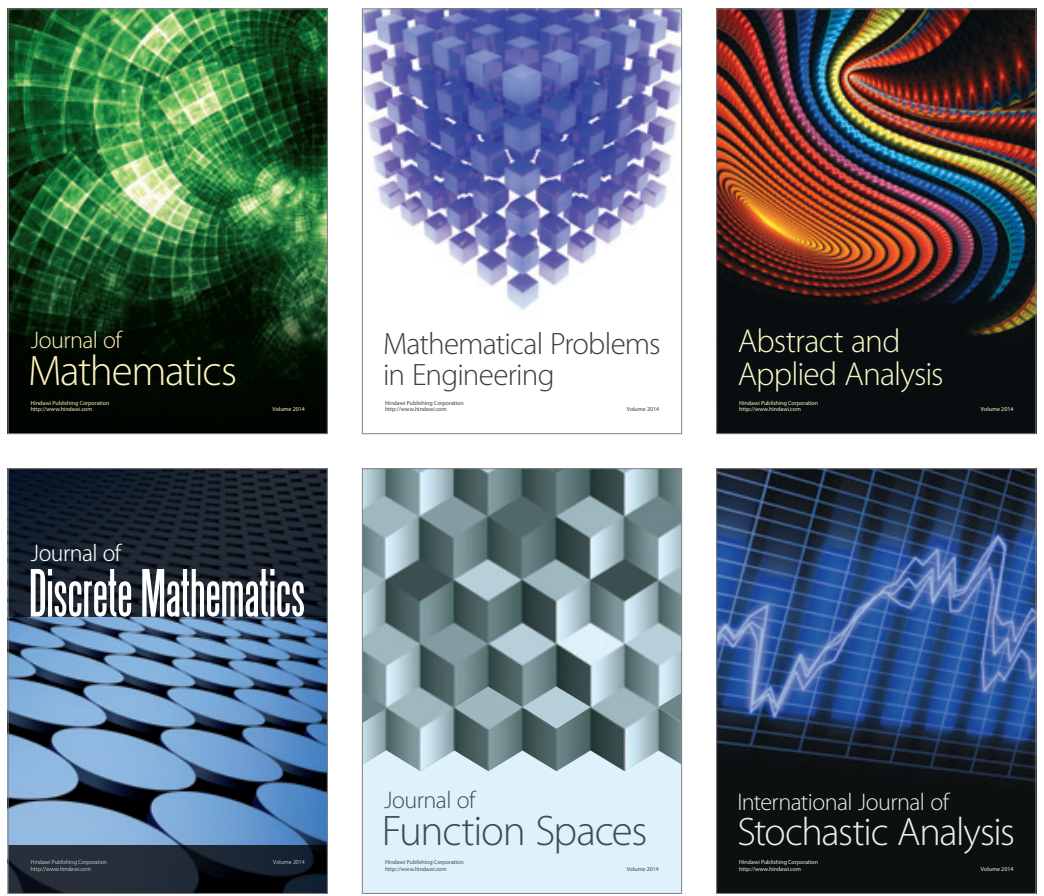

Journal of

Function Spaces

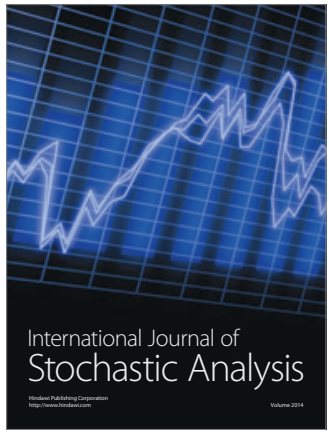

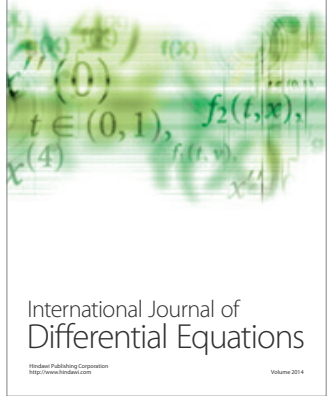
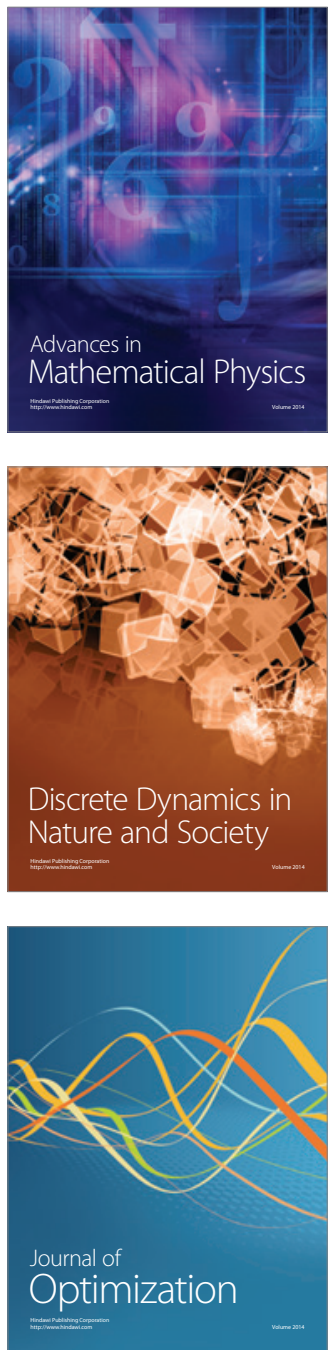